\title{
Características de la intervención psicosocial en el Programa de Desarrollo y Paz del Magdalena Medio como escenario de construcción de paz en Colombia*
}

\author{
[Versión en Castellano]
}

Psycho-social Intervention Characteristics in the Magdalena Medio Peace and Development Programme as a Peace Construction Scene in Colombia

Características da intervenção psicossocial no Programa de Desenvolvimento e Paz do Magdalena Medio como cenário de construção da paz na Colômbia

Recibido el 27 de junio, 2020. Aceptado el 9 de noviembre, 2020

\author{
Francy-Lorena Jaramillo-Grajales** \\ https://orcid.org/0000-0002-4829-2001 \\ Colombia \\ Luisa-Fernanda Cárdenas-Vásquez ${ }^{* * *}$ \\ https://orcid.org/0000-0002-5814-8935 \\ Colombia
}

Para citar este artículo: Jaramillo-Grajales, Francy-Lorena; Cárdenas-Vásquez, Luisa-Fernanda (2021). Características de la intervención psicosocial en el Programa de Desarrolloy Paz del Magdalena Medio como escenario de construcción de paz en Colombia. Ánfora, 28(50), 133-158. https://doi.org/10.30854/anfv28.n50.2021.719 Universidad Autónoma de Manizales. ISSN 0121-6538.

E-ISSN 2248-6941. CC BY-NC-SA 4.0

\section{Resumen}

Objetivos: el artículo busca analizar las características de intervención psicosocial del Programa de Desarrollo y Paz del Magdalena Medio (PDPMM) fundado en 1995 y resaltarlo como una experiencia gestada desde los grupos de

* El contenido del presente artículo es producto de la investigación en la Especialización de Psicología Social Comunitaria y Acción Psicosocial de la Universidad Católica de Pereira. Fecha: julio de 2018 a marzo de 2019. Las autoras declaran que no hubo conflicto de intereses en la ejecución del proyecto de investigación.

** Especialista en Psicología Social Comunitaria y Acción Psicosocial. Licenciada en Etnoeducación y Desarrollo Comunitario. Maestrante en Estudios Culturales. Universidad Católica de Pereira, Risaralda, Colombia. Correo electrónico: francis3482@hotmail.com

*** Especialista en Psicología Social Comunitaria y Acción Psicosocial. Maestrante en Estudios Culturales. Licenciada en Etnoeducación y Desarrollo Comunitario. Universidad Católica de Pereira, Risaralda, Colombia. Correo electrónico: luferk90@gmail.com 
base, develando las formas de participación ciudadana y de organización social. Metodología: la investigación se estructuró bajo un enfoque cualitativo, empleando el método de análisis de contenido, a través del instrumento de la matriz documental. Este proceso metodológico arrojó un análisis en tres momentos: el primero, la caracterización de la fundación del Programa; el segundo, la perspectiva teórica de lo psicosocial y, el tercero, el análisis de las características de intervención psicosocial del Programa de Desarrollo y Paz del Magdalena Medio (PDPMM). Resultados: se encontró que uno de los elementos principales del PDPMM fue definirse desde sus inicios como un proceso social en permanente construcción, cambiante, dinámico y sujeto a una recomposición de sí mismo. Por otro lado, se evidenció que es de vital importancia conocer y validar las experiencias de construcción de paz en Colombia, en este caso particular el PDPMM, que propone desde una metodología participativa, una alternativa para asumir y vivir un territorio, pensando lo regional y resignificando los procesos generados con los actores de la sociedad civil. Conclusiones: se concluye que con el Programa de Desarrollo y Paz del Magdalena Medio los procesos comunitarios fortalecieron la participación, el sujeto político, el tejido social y la construcción de lo público en aras de un bienestar colectivo.

Palabras-clave: Programa de Desarrollo y Paz; Magdalena Medio; Metodología, Intervención psicosocial; Construcción de paz.

\section{Abstract}

Objective: the articleseeks to analyze the psychosocial interventioncharacteristics of the Magdalena Medio Peace and Development Programme (PDPMM) founded in 1995 and highlight it as an experience developed from grassroots groups, revealing manners of citizen participation and social organization. Methodology: the research was structured under a qualitative approach, using the content analysis method, through a documentary matrix instrument. This methodological process yielded an analysis of three moments: first, the characterization of the Program foundation; second, the theoretical perspective of the psychosocial and, third, the analysis of the characteristics of psychosocial intervention of the Magdalena Medio Peace and Development Programme (PDPMM). Results: it was found that one of the main elements of the PDPMM was to define itself from its beginnings as a social process in permanent construction, changing, dynamic and subject to a recomposition of itself. On the other hand, it was evidenced that it is vitally important to know and validate the experiences of peacebuilding in Colombia, in this particular case the PDPMM, which proposes, from a participatory methodology, an alternative to assume and live a territory, thinking about the regional and assign a new meaning to the processes 
generated with the actors of civil society. Conclusions: it is concluded that with the Magdalena Medio Peace and Development Programme, the community processes strengthened elements such as, participation, the political subject, the social fabric, and the construction of the public for the sake of collective well-being.

Keywords: Peace and Development Programme; Magdalena Medio; Methodology, Psychosocial Intervention; Peacebuilding.

\section{Resumo}

Objetivo: 0 artigo busca analisar as características de intervenção psicossocial do Programa de Desenvolvimento e Paz do Magdalena Medio (PDPMM) fundado em 1995 e destacá-lo como uma experiência desenvolvida a partir dos grupos de base, revelando as formas de participação cidadã e organização social. Metodologia: a pesquisa foi estruturada sob uma abordagem qualitativa, utilizando o método de análise de conteúdo, através do instrumento da matriz documental. Esse processo metodológico rendeu uma análise em três momentos: o primeiro, a caracterização da fundação do Programa; a segunda, a perspectiva teórica do psicossocial e, em terceiro lugar, a análise das características de intervenção psicossocial do Programa de Desenvolvimento e Paz do Magdalena Medio (PDPMM). Resultados: verificou-se que um dos principais elementos do PDPMM deveria ser definido desde o início como um processo social em constante construção, mudança, dinâmica e sujeita a uma recomposição de si mesmo. Por outro lado, mostrou-se que é de vital importância conhecer e validar as experiências de construção da paz na Colômbia, neste caso específico o PDPMM, que propõe a partir de uma metodologia participativa, uma alternativa para assumir e viver um território, pensando no regional e resignando os processos gerados com os atores da sociedade civil. Conclusões: conclui que com o Programa de Desenvolvimento e Paz do Magdalena Medio os processos comunitários fortaleceram objetos tais como a participação, o tema político, o tecido social e a construção do público em prol do bem-estar coletivo.

Palavras-chave: Programa de Desenvolvimento e Paz; Magdalena Medio; Metodologia, Intervenção Psicossocial; Construção da paz. 


\section{Introducción}

El presente artículo aborda las características psicosociales que subyacen en la intervención del Programa de Desarrollo y Paz del Magdalena Medio (PDPMM), fundado en 1995 y considerado el primer laboratorio de paz en Colombia; es además reconocido porque logra convocar a la comunidad, entidades públicas, privadas, religiosas y académicas en torno a la transformación comunitaria desde el desarrollo económico, social, político, ambiental y cultural.

En la actual coyuntura política del post-acuerdo de paz en Colombia, es pertinente reconocer las múltiples trayectorias de los procesos de construcción de paz en las comunidades que, en medio de condiciones sociopolíticas complejas, generan una apuesta de reconstrucción del tejido social, asumiéndose como agentes movilizadores de decisiones locales y regionales y articulando los intereses comunitarios e institucionales. Por ello, el propósito de este trabajo se enmarca en reconocer las intervenciones psicosociales en contextos de conflicto armado que logran articularse con instituciones nacionales e internacionales, públicas y privadas, realizando aporte a los procesos de construcción de paz y, a su vez, posicionándose como medio para fortalecer y problematizar las realidades en contextos vulnerados por la violencia.

El análisis busca responder a la pregunta: ¿cuáles son las características de intervención psicosocial en el Programa de Desarrollo y Paz del Magdalena Medio? Esto aportaría a reconocer la importancia de las intervenciones psicosociales como medio para fortalecer y trasformar las realidades en territorios vulnerados por la violencia. Para abordar este interrogante, se tuvo como objetivo principal el análisis de las características de la intervención psicosocial que subyacen en la propuesta del PDPMM. Para dar cumplimiento a este objetivo fue necesario proponer los siguientes objetivos específicos: identificar los fundamentos del Programa y describir las características de intervención psicosocial que subyacen en la metodología del mismo en sus años de creación.

\section{Contexto del Magdalena Medio desde lo geográfico, organizaciones, movimientos sociales y violencia política}

La región del Magdalena Medio se encuentra ubicada entre la Cordillera Oriental y Central, en los departamentos de Antioquia, Bolívar, Boyacá, Cesar y Santander y, en menor medida en Caldas, Cundinamarca y Tolima. Por su geografía estratégica para las ciudades andinas, es considerada una zona de paso al Caribe, Venezuela y a los valles del Sur, permitiendo un desarrollo regional e interdepartamental. Además, se reconoce por sus riquezas naturales como oro, petróleo, fauna, flora y fuentes hídricas, características que permitieron esta- 
blecer una economía extractiva, dando lugar a los primeros asentamientos, los cuales generaron un uso desmedido del medio ambiente, creando escenarios de disputa continua e inequidad para la zona (Observatorio de Paz Integral, 2014).

La importancia geopolítica de la región del Magdalena Medio la ha convertido en uno de los principales focos de la violencia sociopolítica. Dicho escenario, no pudo satisfacer las necesidades básicas de todos los migrantes y pobladores, configurando así las primeras protestas sociales que se valieron de la ausencia del Estado para solucionar conflictos y buscar un bienestar social.

Lo anterior, configuró un territorio con agudos conflictos sociales, políticos, económicos y ambientales, dando lugar al asentamiento de movimientos guerrilleros de la época como el Ejército de Liberación Nacional (ELN), Fuerzas Armadas Revolucionarias de Colombia (FARC) en los años 60, movimientos M-19 y Ejército Popular de Liberación (EPL) en los 70, grupos de narcotraficantes en la década de los 80 y surgimiento de los paramilitares en los 90, convirtiendo al Magdalena Medio en una de las regiones más violentas de Colombia. Tales situaciones, durante años, han afectado directamente a la población, vulnerando sus derechos humanos y afectando los procesos de trabajo social y comunitario de los líderes y organizaciones sociales, movimientos sindicales, entre otras.

En la década de los 90, en el Magdalena Medio hacen presencia varias iniciativas de la sociedad civil, dando cuenta del agenciamiento de las comunidades en sus capacidades para reconocer y comprender el conflicto, así como para trabajar de manera colaborativa, con las organizaciones no gubernamentales, movimientos políticos nacionales y de iglesias católicas. Entre las organizaciones presentes en esta dinámica, se encuentran las de los pobladores, que se caracterizan por ser agrupaciones informales para la distribución del espacio de trabajo y formas asociativas de producción, en las cuales las relaciones de comunicación son horizontales, lo que implica la organización y distribución del territorio mediante acuerdos. Este tipo de organización de base de mineros, pescadores o agricultores, depende de valores como la equidad, la solidaridad, el valor de la palabra y la unidad de propósitos (Programa de las Naciones Unidas para el Desarrollo [PNUD], 2008, p. 99).

Así mismo, se encuentran las organizaciones para la lucha reivindicativa que se identifican como movimientos dirigidos a defender la posesión de la tierra, a exigir el respeto por la vida y a mejorar sus condiciones. Un ejemplo de este tipo de agrupación en la región es la Mesa Campesina del Éxodo, que en 1998 movilizó miles de campesinos para denunciar la arremetida paramilitar y las violaciones de derechos humanos por parte del Estado (PNUD, 2008, p. 99). También están las organizaciones para la asistencia directa en las que las acciones colectivas brindan atención focalizada a grupos especialmente vulnerables, como la tercera edad, madres cabezas de hogar, niñas y niños, población juvenil, 
entre otras, a partir de la financiación e iniciativas regionales o nacionales que, si bien cumplen un papel complementario, en ocasiones cubren responsabilidades del Estado.

Para dicho territorio, la historia de los movimientos sociales y de las organizaciones da cuenta de una dinámica significativa, diversa y sólida que ha logrado, frente a los intereses colectivos, generar acciones que promuevan otras realidades a partir de la puesta en conversación de los actores tanto de lo público como de lo privado; se destaca la presencia de la Pastoral Social de la Iglesia Católica, la Corporación Regional de Derechos Humanos (CREDHOS), la Organización Femenina Popular de Barrancabermeja, la Asociación de Trabajadores Campesinos del Carare (ATCC), la Asociación Campesina del Valle del Río Cimitarra (ACVC), la Federación Agrominera del Sur de Bolívar (FEDEAGROMISBOL) y la Unión Sindical Obrera (USO) (PNUD, 2008, p.17).

Después de reconocer las características del territorio del Magdalena Medio y evidenciando las tensiones que históricamente lo han configurado, es necesario comprender los fundamentos iniciales sobre los cuales se construyó el Programa de Desarrollo y Paz, reconociendo sus líneas de intervención frente a los Derechos Humanos, procesos sociales y productivos, siendo el escenario de intervención psicosocial sobre el cual se realizó esta investigación.

\section{Surgimiento del Programa de Desarrollo y Paz del Magdalena Medio}

En el Magdalena Medio en Colombia, líderes jesuitas como el padre Francisco de Roux, le apostaron a construir una sociedad más equitativa, justa y garante de derechos. Así, el 17 de octubre de 1995 se constituyó el Consorcio Centro de investigación y Educación Popular-Sociedad Económica de Amigos del País (CINEP-SEAP) que realizó el diagnóstico regional con la ayuda de la Diócesis de Barrancabermeja y propuso la creación del Programa Desarrollo y Paz del Magdalena Medio (PDPMM), como instrumento capaz de insertarse en la vida del territorio y generar dinámicas favorables al desarrollo y la paz (Red Nacional de Programas Regionales de Desarrollo y Paz [Redprodepaz], 2016).

Esta iniciativa permitió reconocer las potencialidades de las comunidades y la garantía de la dignidad para todos y todas. Después de realizar un diagnóstico de la región De Roux (1999), el programa se fijó como objetivos: a) El desarrollo económico debe ir en consonancia con un mejoramiento de la dignidad y calidad de vida, b) Propender por la sostenibilidad ecológica, económica y social como elementos centrales de la estrategia, c) Construir una democracia participativa y una consolidación del espacio público (p. 20). 
El análisis de fondo que sustentó esta ruta fueron dos cuestionamientos: el primero, ¿cómo es posible que una región tan rica como la del Magdalena Medio (por sus habitantes, el petróleo, las minas de oro, la biodiversidad) pueda vivir con municipios y veredas en donde la pobreza alcanza niveles muy altos? Y el segundo, ¿cómo es posible que un pueblo alegre, trabajador, soñador, viva una situación de tanta violencia? Por ello, dentro de lo criterios orientadores que priorizaron las acciones y proyectos del PDPMM se expresaron los siguientes: "Primero la vida", el respeto de la dignidad humana, la construcción de la región entre todos y todas, el desarrollo para la paz sostenible, la solidaridad con la población víctima y vulnerable, el reconocimiento y valoración de la diferenciación y promoción del sujeto social y de lo público (Vargas et al., 2011, p. 5).

En la realidad colombiana, la construcción de paz se convirtió en el campo de participación para muchos actores que, desde múltiples experiencias y comprensiones, han fortalecido el marco epistémico a partir del cual se intervienen las realidades en clave de paz y de escenarios transicionales. Sin embargo, las formas particulares y contextuales de persistir en medio del conflicto armado, pueden ampliar la mirada de lo complejo que es enunciar y hacer experiencia la paz, mirada que se asume en adelante como las acciones encaminadas principalmente a posibilitar la participación política y democrática, ofrecer justicia para los responsables de los hechos y establecer los pilares de la reconciliación desde cambios estructurales que establezcan garantías de no repetición (Rodríguez, 2017).

\section{Lineas estratégicas del Programa de Desarrollo y Paz del Magdalena Medio en Colombia}

El PDPMM promueve valores como la paz, la vida, la productividad, la aceptación de la diferencia, la solidaridad y pretende fortalecer capacidades en sus pobladores como la asociación, creación de organización, la toma de decisiones, entre otras; a lo largo de su historia ha orientado su quehacer en tres líneas estratégicas ${ }^{1}$, que interactúan entretejidas, son interdependientes e integrales en el territorio, a partir del desarrollo de las acciones con las comunidades, organizaciones y pobladores de la región. Estas son: 1. Derechos Humanos, Diálogo y Construcción de Paz, 2. Procesos Sociales, Culturales y de Gobernabilidad Democrática, 3. Procesos productivos y ambientales para la Equidad y el Desarrollo Sostenible.

1. En este link se amplía información http://www.pdpmm.org.co/index.php/el-programa 
El PDPMM se posicionó como el primer proceso regional nuevo y diferencial en el que, más allá de movimientos políticos y culturales, el grupo creciente de pobladores del territorio, de cara a su compleja realidad política y social, avanza hacia la paz desde la protección de la vida (De Roux, 1999, p. 15). Esta propuesta regional se consolidó como el primer Laboratorio de Paz en Colombia, por medio de un proceso metodológico flexible y ajustable a las necesidades territoriales, que dio cuenta de lo que sucedía como un entramado de vivencias, significaciones y dinámicas.

Frente a este contexto, las intervenciones que se han realizado en Colombia para acompañar a las poblaciones víctimas del conflicto armado están enmarcadas tanto desde las instituciones estatales como desde las organizaciones de base, impactando de forma distinta los grupos poblacionales que intervienen pero, a la vez, movilizando a la creación de espacios de reconciliación. Por lo tanto, los procesos de intervención deben enraizarse en formas de grupalidad y dialogicidad, sin separarlas de los desdoblamientos individuales que forman y exigen nuevos núcleos de producción de esa subjetividad social a nivel institucional, es decir, en la familia, la escuela, la organización laboral y la comunidad (González, 2004, p. 24).

En ese sentido, es necesario resaltar que durante las últimas tres décadas los marcos de referencia y de interpretación en construcción de paz en el país, se han fortalecido en diferentes sectores, haciéndose visibles los laboratorios de paz como formas propias y contextuales de responder y tramitar las violencias en los cuerpos y los territorios.

Al respecto Barreto (2015) en el artículo titulado El Programa de Desarrollo y Paz del Magdalena Medio, ¿̇U modelo de construcción de paz para el postconflicto en Colombia? reconoce que el PDPMM "se configura como un modelo consolidado y válido de construcción de paz positiva a nivel regional, de lo cual se pueden rescatar diversas enseñanzas y ‘buenas prácticas’ para la construcción de una paz sostenible y duradera en Colombia” (p. 462).

Desde esta perspectiva se evidencian las agencias con apuestas locales y regionales como una construcción que transita, en medio de las dificultades, sobre las alternativas de trasformación que garanticen la vida en los territorios. Esto, visibiliza las búsquedas que van más allá de la simple ausencia del conflicto, rescatando en la cotidianidad, las particularidades, estrategias y alianzas entre sujetos, organizaciones y localidades como enclave significativo para problematizar dicho escenario coyuntural en nuestra realidad.

Otro aporte lo hace Saavedra (2006) en el artículo El programa de Desarrollo y Paz del Magdalena Medio y la Red Prodepaz da luces sobre como el PDPMM ha buscado establecer vínculos y acciones a partir de la lectura compleja de actores, intereses, conflictos, identidades, y diferencias, desde posicionamientos críticos y 
propositivos; ha permitido ello construir sinergias entre lo público y lo privado, reconfigurando y evidenciando la porosidad que representa la institucionalidad y amplía el posicionamiento de lo público de los sujetos. Son lecturas que invitan a leer las comprensiones, posicionamientos y metodologías que han significado el PDPMM como construcción que por décadas ha buscado reconfigurar las líneas de intervención social y político en contextos vulnerados.

Ahora de forma particular, se revisa desde donde se asume la perspectiva teórica de lo psicosocial como fundamento a la presente investigación, reconociendo el impacto que tienen las intervenciones psicosociales como apuestas para pensar la relación de lo individual y lo colectivo en la trasformación comunitaria.

\section{Perspectiva teórica de lo psicosocial}

Los cambios sociales, culturales y políticos que emergen en las sociedades actuales conducen a crear nuevas formas de asumir y enfrentar los desafíos, conflictos y posibilidades, algo que no puede darse adaptando modelos de intervención europeos o norteamericanos para intervenir fenómenos sociales latinoamericanos. Por eso, es necesario implementar intervenciones sociales contextualizadas, que permitan crear nuevas realidades desde y para una comunidad específica.

La importancia de la discusión en torno a lo psicosocial debe conducir al reconocimiento de una categoría en medio de una tradición académica, que enuncie las múltiples posibilidades de análisis en las cuales trascienda la descripción solo de lo individual y social, dado que la condición humana no solo es resuelta desde está reducción, pues también está atravesado por estructuras de poder tanto en lo endógeno como en lo exógeno. El ser humano debe analizarse a partir de lo psicológico, pero ligado a su realidad socio-histórica y contextual, pues no es preciso verlo en una sola dimensión; al ser un actor social está inmerso en una interacción simbólica: individuo, familia - grupo y comunidad (Corporación Juan Bosco, 2007).

Lo psicosocial es una perspectiva para comprender e intervenir la realidad sin reducirla a procesos psíquicos o sociales excluyentes entre sí. En los aportes de Díaz y Díaz (2015), se sugiere un acercamiento al hombre concreto, para detallar las características individuales y grupales de un contexto específico, permitiendo abordar de manera integral posibles alternativas de cambio (p. 63).

Para las instituciones públicas, lo psicosocial se toma como "una forma de entender las respuestas y los comportamientos de las personas en un contexto cultural, político, económico, religioso y social determinado" (Bello, 2004, p. 192). La clave está en cómo comprender estos procesos que emergen y están en 
movimiento, razón por la cual son una nueva condición de la realidad, dado que aborda tanto las subjetividades individuales como las identidades colectivas.

Los seres humanos se construyen con los otros y desde lo otro, en busca de unas habilidades sociales, comunicativas y simbólicas, lo que implica la emergencia de la propia subjetividad personal y la construcción o reconstrucción de la colectividad (Carmona, 2009, p. 53). Por su parte, Villa (2012) afirma que hablar de "lo psicosocial implica, por lo tanto, una posición que atraviesa no solo lo teórico y la intervención misma, sino un lugar ontológico, ético - político, epistemológico y metodológico” (p. 353).

Así, lo psicosocial es una forma de analizar las problemáticas y fenómenos sociales, teniendo como objeto las interacciones que se dan en un medio social; aunque ninguna disciplina en particular se ha dedicado a hacer un estudio riguroso sobre esta categoría, algunos intelectuales latinoamericanos coinciden en que no es posible hablar del ser humano sin tener en cuenta su contexto, siendo este un espacio simbólico y físico, que crea significaciones en los sujetos que lo habitan, desarrollando competencias y habilidades que reconocen la diversidad de escenarios y de pensamientos, los cuales configuran y reconstruyen los tejidos sociales; esto último, solo ocurre a través de las experiencias personales, colectivas y en las relaciones cotidianas.

Reconociendo ya la perspectiva de lo psicosocial, es preciso dar cuenta de estas tres teorías latinoamericanas: la psicología social comunitaria, la psicología social crítica y la psicología de la liberación, que han permitido crear enfoques sobre los cuales se asume la intervención psicosocial para problematizar las realidades, los sujetos y las formas de intervención institucional.

\section{Perspectiva teórica de la relación entre Psicología social comunitaria, Psicologia crítica y Psicologia de la liberación}

Desde Montero (2004), "el qué hacer, cómo hacer y el pensar psicológico desarrollados en el campo social latinoamericano a finales del siglo XX, y lo que va del siglo XXI, se han configurado como una respuesta alternativa e innovadora a las necesidades del contexto, haciendo sus aportes a los múltiples cambios de pensamientos, gestados ante las crisis de los modelos tradicionales en el campo de las Ciencias Sociales” (p. 17). Dentro de estos campos, se tiene a la Psicología Social Comunitaria, la Psicología de la Liberación y la Psicología Social Crítica, corrientes de conocimientos que tienen relaciones, semejanzas y diferencias entre sí, aunque mantengan su interés por el estudio de las dinámicas socioculturales del continente latinoamericano donde han asumido su identidad y sus raíces. 
La psicología comunitaria, se definió en sus inicios como el estudio de los factores psicosociales que permiten desarrollar, fomentar y mantener el control y el poder que los individuos pueden ejercer sobre su ambiente natural y social, para solucionar problemas que los aquejan y lograr cambios en esos ambientes (Montero, 1984, p. 390). En esta se configura la participación desde la movilización de las comunidades, grupos o personas en su accionar, en su reconocimiento como actores sociales que reconstruyen sus realidades particulares.

Por su parte, la corriente crítica se asume en un discurso político que, desde el análisis consiente de los ejercicios positivistas, evidencia las condiciones socioeconómicas y su efecto sobre el campo de la ciencia y las formas de asumir los fenómenos sociales; así mismo, busca generar reflexión y compromiso con el otro, dando cuenta de la complejidad que implican las realidades y generando posibilidades de cambio.

La crítica subvierte el modo de ver las cosas; desencaja los mecanismos de poder que sostienen posiciones establecidas y abre nuevas perspectivas al conocimiento. Ella es parte de la complejidad del mundo, usualmente tan difícil de aceptar (Montero 2004, p. 19). Su importancia radica en que es una herramienta para encontrar diversas formas de asumir lo social, priorizando la diversidad de los actores sociales y la relación entre los fenómenos y el contexto en el que se desarrollan, tomando la complejidad de dicho escenario de construcción e invitando a su estudio desde una perspectiva holista.

Por su parte, la psicología de la liberación define su accionar al trabajar con las víctimas de opresión, siendo estos los sujetos que han sido excluidos de los bienes sociales y de la toma de decisiones en lo público. La liberación es, entonces, la re-significación de las capacidades y potencialidades que dichos sujetos tienen y que han sido negados en marcos históricos, culturales y sociales de poder y segregación, asumiéndolos como sujetos activos en su trasegar a otras realidades. Para Martin Baró (1986), "la función de la psicología no sería, por tanto, la de predecir y controlar la conducta, y mucho menos la de pretender ofrecer soluciones fáciles a los dilemas de la existencia, sino "liberar" al ser humano, brindándole mayor autonomía y posibilidades reales de elección, superando esquemas alienados de conducta” (Dobles, 2009, p. 7).

Estas tres tendencias dan forma a un camino particular de hacer psicología, expresando sus valiosos hallazgos en la complejidad y dinamismo de lo social, demostrando que los tejidos sociales que se construyen en una comunidad, trasforman el entorno tanto de los sujetos de intervención, como del rol desempeñado por las intervenciones de los profesionales.

En la presente investigación se destacan algunos elementos de análisis sobre cómo se trasforma un contexto a partir de las interacciones entre las organizaciones de base, las instituciones públicas, la academia y la iglesia católica, en 
medio de un contexto históricamente vulnerado y en el que se confrontan los ideales, los intereses particulares y las metodologías de intervención para significar la construcción de lo colectivo como modelo de desarrollo regional.

\section{Metodología}

La investigación se estructuró bajo un enfoque cualitativo que, como lo afirman Rodríguez, Gil y García (1996), "la investigación cualitativa estudia la realidad en un contexto tal y como sucede, tomando como eje central los significados y sentidos que se construyen a través de las relaciones entre las personas" (p.10). Se empleó como método el análisis de contenido, que desde la perspectiva de Abela (2002) "se define como la interpretación de textos ya sean escritos... u otra forma diferente donde puedan existir toda clase de registros de datos (...) se basa en la lectura (textual o visual) como instrumento de recogida de información" (p. 2).

Para este análisis de contenido, se utilizó como instrumento una matriz de análisis documental que consiste, según Finol y Nava (1999), en organizar los datos conceptuales necesarios para el análisis, de donde se derivan categorías y subcategorías que soportan la discusión teórica.

El proceso metodológico consta de tres etapas y se desagregan en siete pasos a saber de la siguiente manera:

\section{Etapa I. Identificación de fuentes documentales}

\section{Paso 1: Consultas bibliográficas virtuales.}

Se realizaron consultas bibliográficas virtuales y presenciales, que arrojaron documentos referidos y sistematizaciones. Se encontraron gran variedad como: libros 5 , páginas web 6 , artículos 12 , sistematizaciones 5 , informes de gestión y de evaluación 5; esto, permitió hacer una selección de documentos pertinentes a la investigación dentro de los que se pueden mencionar varios documentos como el "Documento central de diagnóstico, conclusiones y recomendaciones, Programa de Desarrollo y Paz en el Magdalena Medio, PDPMM" (De Roux, 1996), "El proceso metodológico del PDPMM" (Katz, 1999b). "El Magdalena Medio en medio del conflicto y de la esperanza" (De Roux, 1996), "Rutas y vivencias de nuestra gente. Sistematización de experiencias en los procesos de los pobladores y pobladoras del Magdalena Medio" (PNUD, 2008), "La construcción de lo público en el escenario actual del Magdalena Medio" (Katz, 1999a), "Los proyectos del PDPMM, un laboratorio de paz" (Moncayo, 1999). 


\section{Paso 2: Consultas bibliotecas.}

Se hizo necesario una búsqueda directa en las bibliotecas del Banco de la República y la Biblioteca Pública Román Correa Mejía en la ciudad de Pereira, el Centro de Investigación y Educación Popular (CINEP) y la Redprodepaz (Bogotá) que en los últimos años hacen sistematizaciones y acompañamiento de los procesos de construcción de paz en Colombia.

\section{Paso 3: Focalización CINEP-REPRODEPAZ.}

Se realizó un acercamiento a los catálogos virtuales del CINEP y Redprodepaz, en la búsqueda de acceso a textos fundacionales vitales para identificar los fundamentos, metodologías y resultados del PDPMM.

\section{Etapa II. Elaboración de instrumentos y recolección de información}

\section{Paso 4: Revisión de textos con mirada a profundidad.}

Permitió reconocer los discursos de la comunidad, los profesionales y las instituciones públicas y privadas que participaron en la construcción del Programa.

Paso 5: Elaboración de matriz de análisis documental.

Para sistematizar la información se procedió a la elaboración de una matriz de análisis documental estructurada en siete columnas (tipo de texto, título de texto, autor, año, ideas de intervención social desde PDPMM, ideas características intervención psicosocial, categorías emergentes).

\section{Paso 6: análisis de textos y categorias emergentes.}

La lectura a profundidad que permitió realizar el cruce de los referentes teóricos asumidos en la investigación con los hallazgos empíricos desde el análisis de los textos, de los cuales fueron emergiendo categorías y subcategorías que le apuntan a posicionar y enunciar las características de la intervención psicosocial encontrados en el PDPMM. A continuación se presenta una tabla que permite observar las categorías y las subcategorías, las cuales se agruparon dando fundamento a los cuatro apartados del análisis de esta investigación. 
Tabla 1. Categorías y subcategorías que emergen en esta investigación

\begin{tabular}{|c|c|}
\hline CATEGORÍA & SUBCATEGORÍAS \\
\hline Intervención comunitaria & $\begin{array}{c}\text { - Conflicto sociopolítico } \\
\text { - Diagnóstico participativo } \\
\text { - Fortalecimiento ciudadano (sujeto } \\
\text { político) }\end{array}$ \\
\hline Construcción local y regional & $\begin{array}{c}\text { - Estrategias de proyectos subregio- } \\
\text { nales } \\
\text { - Participación comunitaria } \\
\text { - Tejido social }\end{array}$ \\
\hline $\begin{array}{c}\text { Intervención psicosocial en el } \\
\text { PDPMM }\end{array}$ & $\begin{array}{l}\text { - Fortalecimiento institucional } \\
\text { - Construcción de lo público } \\
\text { - Transformación comunitaria }\end{array}$ \\
\hline
\end{tabular}

Fuente: elaboración propia

\section{Etapa III. Análisis de la información}

Paso 7: Interpretación de información.

El proceso que se adelantó, como ejercicio situado, permitió el diálogo entre la especificidad generada en las intervenciones de los años de existencia del PDPMM, las lecturas conceptuales y las agencias mismas de las sujetas investigadoras, relacionamiento que genero el análisis y la discusión teórica. Con todo esto, se buscó aportar a la creación de significados y sentidos en la construcción de paz desde la mirada de lo psicosocial, apuntando a que la información, no solo sea recolección de datos, sino, que permita crear e interpelar los lugares de enunciación que se movilizan en esta realidad (Díaz, González-Rey y Arias, 2017).

A continuación, se centrará la discusión teórica en las características de intervención psicosocial que subyacen en el Programa de Desarrollo y Paz, las cuales se abordarán por medio de tres líneas de análisis que integran las categorías y subcategorías. 


\section{Resultados}

\section{Intervención comunitaria}

El Magdalena Medio es una región históricamente afectada por el conflicto armado, en la que han hecho presencia diferentes grupos insurgentes con intereses particulares (drogas ilícitas, extracción minera, corredores estratégicos), que han impactado negativamente a las comunidades con ataques, secuestros, desplazamientos, homicidios, infiltraciones, entre otros; ello ha aumentado los niveles de pobreza y exclusión, impidiendo que se gestaran procesos comunitarios con justicia social. No obstante, el Programa se consolidó en sus inicios como "una apuesta desde la sociedad civil, desde el territorio y sus pobladores, a pesar de sus limitaciones, han logrado convocar a sectores históricamente opuestos y excluidos a poner sus intereses en la agenda pública" (Guerrero et al., 2011, p. 14).

Dado este contexto, el Programa de Desarrollo y Paz constituye un Espacio Humanitario (EH) en el Magdalena Medio ya que, como proceso social, ha declarado autonomía frente a los actores del conflicto armado, con el objetivo de permanecer en su territorio y encaminado a forjar un escenario de bienestar común y de construcción de paz. Estos EH están soportados por el Derecho Internacional Humanitario (DIH) que protege a la población no combatiente en áreas especiales para salvaguardarla de los efectos de la guerra o de los conflictos armados internos.

Desde un enfoque psicosocial, el hecho de que las comunidades con el paso del tiempo, tomen decisiones organizativas, y visibilicen por medio de la comprensión del conflicto sus debilidades y fortalezas para aprovechar el acompañamiento de las organizaciones gubernamentales y no gubernamentales, es un paso estratégico para pensar lo dinámico y contextual que deben ser las intervenciones, pues no se trata de quitar responsabilidades al Estado, sino por el contrario, reconocer su función en la articulación con los derechos de las poblaciones. En este sentido, Vargas (2010) hace referencia en que "la sociedad civil fortalece los derechos humanos, los divulga, re-significa el tema de la ciudadanía, es decir, los pobladores como sujetos de derechos convocan a los grupos armados, alcanzando acuerdos humanitarios, locales y coyunturales" (p. 11).

Tales aspectos posibilitan una construcción mediada por los intereses de todos los sectores de la sociedad, en aras de crear espacios de desarrollo local y regional; por eso, intervenir en contextos multi-problemáticos implica un compromiso con los problemas sociales y con sujetos particulares donde se dispone apostar y mediar en realidades diversas, pues son muchos los actores sociales que hacen presencia en un mismo territorio. Desde allí, la construcción de ciu- 
dadanía supone un desafío, en tanto se pretende movilizar nuevas subjetividades para gestar procesos de justicia, equidad, sana convivencia y paz.

Desde esta investigación, se toma la participación como elemento clave para la intervención comunitaria, resaltando su efecto político en relación con la formación de ciudadanía para el fortalecimiento de la sociedad civil y a través de esta, se genera un empoderamiento para la construcción del sujeto político, de acuerdo con Montero (2004), "la participación comunitaria es un proceso organizado, colectivo, libre, incluyente en el cual hay una variedad de actores, actividades y de grados de compromiso que está orientado por valores y objetivos compartidos, en cuya constitución se producen trasformaciones comunitarias e individuales" (p. 109).

De esta manera, al participar se incluyen a todos los actores de dicho proceso, reconociendo los agentes internos y externos del mismo; por ello, se parte del escenario del PDPMM como proceso de participación colectiva que devela los esfuerzos y las apuestas comunes instauradas bajo una mirada integral que incluyó a sus pobladores, partiendo de la consigna de la protección a la vida y al desarrollo regional. "El trabajo se fue construyendo desde la familia, la finca campesina, las veredas y las organizaciones, integrando y compartiendo puntos de vista con la historia y sus memorias, a fin de apoyar así, la configuración del proyecto colectivo" (PNUD, 2008, p. 84).

No obstante, lo enunciado anteriormente está marcado por tensiones, por saberes en disputa y por intereses individuales, que configuran diversas representaciones de lo colectivo y que también permite comprender que la intervención psicosocial no es una acción perfecta, sino dinámica que incluye en su acción encuentro y desencuentros, vistos como oportunidad para re direccionar de nuevo.

Por su parte, el proceso de diagnóstico del PDPMM articuló a más de dos mil personas y a diferentes instituciones que estuvieron vinculadas de diversas formas, así como un grupo significativo de organizaciones como: la Coordinadora Regional de Derechos Humanos CREDHOS, la Organización Femenina Popular OFP, la Coordinadora Popular de Barrancabermeja, las Cámaras de Comercio, el Comité Gremial, la Uso Regional y Nacional, las Asociaciones de Pescadores, el Grupo Gestor del Puerto Petrolero, el Equipo de Relaciones Externas de Ecopetrol, la Asociación de Campesinos de la Cuenca del Río Cimitarra, la Asociación de Frijoleros de Santa Rosa ASOCALIMA, el Centro CLEBER de Simití, CORMAGDALENA, FUNDESMAG, las universidades locales INUPAZ, UCC y UIS, la Brigada de Paz y la Cruz Roja Internacional, la Pastoral Social de la Diócesis y el Servicio Jesuita de Desplazados.

El diagnóstico se llevó a cabo con los pobladores entre el año 1995 y 1996 en diferentes municipios de la región, con el objetivo de visibilizar las experien- 
cias individuales y colectivas, dificultades y fortalezas del territorio, para consolidar un enfoque metodológico flexible y ajustable. Según de Roux (1999) "el proceso metodológico del Programa da cuenta de unas variables fundamentales para pensar un desarrollo integral: región, poblamiento, cultura, economía rural y urbana, petróleo, instituciones, política local, educación y medio ambiente" (p. 19). Desde una perspectiva psicosocial, las diversas articulaciones entre pobladores, asociaciones e instituciones gubernamentales no solo dan cuenta de objetivos comunes, sino también de unos lugares de mediación, los cuales develan que son fundamentales los tiempos y los espacios para llegar a construirse acuerdos comunitarios y no decisiones asistenciales en la intervención psicosocial.

Lo anterior, dio paso al objetivo del Programa que hizo énfasis en la convivencia ciudadana para una cultura de la paz que sentara bases en la construcción del espacio público, donde nadie sea excluido, en palabras de De Roux (1999),

Se buscaba la puesta en marcha de una economía sostenible, desarrollada y controlada por pobladores, organizaciones campesinas y empresarios locales, que pusiera la calidad de vida regional como primer objetivo de la actividad productiva, y por un Estado y una sociedad que forjaran el capital social y natural indispensable para la convivencia y las iniciativas de los grupos (p. 20).

Se comprende desde esta dinámica la importancia de construir puentes de trabajo y cooperación entre el Estado, instituciones y gremios académicos, pues son precisamente los procesos gestados desde la sociedad civil en su lucha por una vida digna los que construyen y trasforman las prácticas ejercidas en sus territorios. En medio de la coyuntura sociopolítica en la que se pensó el Programa, se partió de las necesidades de la comunidad para priorizar a las víctimas del conflicto armado hacia el empoderamiento de niños, niñas, jóvenes, mujeres, comunidades, como actores sociales y políticos fundamentales para el fortalecimiento ciudadano.

Los procesos comunitarios del PDPMM se comprenden como un proceso comunitario que resalta la construcción de lo colectivo, donde se movilizan los grupos de base que, en articulación con otras instituciones públicas y privadas, sobrepasan las prácticas asistencialistas y momentáneas a las que están acostumbradas y perfiladas algunas comunidades colombianas. El acercamiento al Programa evidencia cómo los procesos de intervención comunitaria requieren tiempo y espacio; de esta forma, desde el año 1995 se están construyendo no solo bajo un plan de gobierno sino como proyecto regional, una trasformación cultural, socio-política y económica para forjar territorios de paz.

Así, el proceso metodológico presenta un ejercicio que particulariza y posiciona al municipio como primer escenario de planeación, desde la participación 
y concertación, pero que sistemáticamente se incluye en una dinámica regional proyectada a un futuro colectivo, como se verá en el siguiente apartado.

\section{Construcción local y regional}

Asumir y sostener un proceso metodológico integrador en el amplio contexto del Magdalena Medio, fue siempre uno de los principales retos del Programa, por lo que se consideró necesario trabajar bajo lógicas descentralizadas pero vinculadas, constituyendo así, un punto de encuentro regional en Barrancabermeja y seis delegaciones territoriales, representando las subregiones, conformadas por los 29 municipios participantes.

La creación de propuestas contextualizadas en cada subregión, se direccionaron en una lógica integradora para el Magdalena Medio, las cuales buscaron respaldo y legitimación institucional a través de pactos municipales. En medio de su desarrollo y producto de su permanente evaluación se demostró que dichos proyectos subregionales construidos inicialmente, no se ajustaban a la realidad del diagnóstico realizado, sino más bien a propuestas en el marco institucional establecidas por expertos académicos y que no evidenciaban una construcción social para el desarrollo regional, pues debían recoger las ideas de los pobladores; según De Roux (1999), “tenía que ser algo propuesto por la misma gente y en coherencia con sus dinámicas sociopolíticas, algo que ellos presentaban a la sociedad mayor y al Estado, a la financiación externa o interna” (p. 24).

Lo anterior, llevó a abandonar los proyectos subregionales apostándole a una propuesta de carácter regional y de asociación de municipios, que llegaría a posicionar al Programa el estatus de un modelo de desarrollo. Estas propuestas creadas desde mayo de 1997 hasta mayo de 1998, constituyeron visiones compartidas sobre las posibilidades para asegurar la convivencia y la superación de la pobreza de manera sostenible; por lo tanto, y como referentes de futuro, se proyectaron a diez años buscando la sostenibilidad y pertinencia para los contextos y sus realidades, soportadas sobre consideraciones de carácter social, colectivo y humanitario.

Este aspecto es fundamental, pues deja comprender, además, cómo los profesionales que realizan las intervenciones psicosociales deben tener una postura ético política, que implica reflexionar los objetivos establecidos desde la institucionalidad (plan, programa o proyecto) en aras de mitigar la acción con daño que esta pueda generar y, a partir de las prácticas contextuales, identificar vínculos y actores que se convierten en guías para movilizar acciones creadas por y para ellos mismos.

En la transformación social la participación de las personas que conforman una comunidad es un elemento indispensable, pues son ellas las que deben lide- 
rar, ejecutar y evaluar los impactos, con el acompañamiento de los profesionales psicosociales, como hace mención De Roux (1999) “el elemento participativo en el PDPMM, hace que la aspiración formulada como futuro posible y como compromiso de personas, grupos e instituciones, salga de los pobladores y desde el inicio involucre a la gente en su maduración y ejecución.” (p. 24).

Así, desde una perspectiva de intervención psicosocial, se consideran tres acciones que posibilitan la intervención psicosocial: la primera, es la lectura del contexto, en la que es necesario participar para reconocer las dinámicas que lo caracterizan y le dan sentido. La segunda, es la creación conjunta de estrategias con la comunidad, generando posibilidades de cambio desde la participación colectiva, dejando capacidades instaladas en los contextos. La tercera, es la generación de puentes institucionales, agenciando el acceso a la oferta gubernamental, como garantes y promotores de los derechos ciudadanos y públicos. Así, quien interviene tiene consigo la tarea de verse a sí mismo como un sujeto en permanente formación, donde la relación sujeto-sujeto va a brindar la posibilidad de generar un aprendizaje en espiral que permita la consciencia crítica como sujetos políticos que transforman sus realidades y fortalecen el tejido social.

La construcción de lo local a lo regional da cuenta de un ejercicio complejo y dinámico, que dio solidez a los esfuerzos concretos de municipios y subregiones, uniendo esfuerzos para construir una región social, cultural y políticamente inclusiva. En el campo de la Psicología Social, estas apuestas se asumen como una apertura para su resignificación política y para su comprensión desde la interdisciplinariedad, puesto que dichos escenarios de construcción demuestran la creación, no solo de un marco organizativo para el beneficio de todos, sino también la apropiación de discursos y de prácticas alternativas que dignifican la vida.

Estas iniciativas comunitarias movilizan recursos colectivos, a través de la participación para el logro de objetivos, desde el proceso metodológico del PDPMM que desea impactar, según Katz (1999a), en "la construcción del tejido social, en la medida en que promueve bases de consenso entre los pobladores, alrededor de la definición de un futuro que responda de manera equitativa a las necesidades e intereses presentes en la vida de la región” (p. 77).

La metodología de trabajo propuesta del PDPMM visibiliza ejercicios que buscan transformar las dinámicas contraproducentes y proponer puntos de negociación con los actores de la región, asumiendo la diferencia como un elemento potencializador para la consolidación de dinámicas que contribuyan al desarrollo integral. Por ello, a continuación se resaltan unos aportes estrategias en la construcción metodológica del Programa, visibilizando su enfoque de intervención psicosocial y que son una apuesta para el fortalecimiento territorial e institucional, el desarrollo humano y económico en vía de la reconstrucción del tejido social. 


\section{Intervención psicosocial en el PDPMM}

El PDPMM propuso en sus años fundacionales un modelo de intervención social que respondiera a dos escenarios posibles para lograr un desarrollo integral, según Katz (1999a) "el primero; el fortalecimiento institucional del Estado desde la participación activa de la sociedad civil, y el segundo; un desarrollo humano y económico integral en donde se reconozca conocimientos, intereses y experiencias de vida de los pobladores" (p. 77). La integralidad supone también un desarrollo económico, destinado a una construcción sostenible, donde se reconozca los aportes de las prácticas productivas de los pobladores, como lo menciona Vargas (2010),

La propuesta busca demostrar que es posible un campesinado empresarial que mantenga la seguridad alimentaria, proteja y reproduzca las tradiciones culturales, el arraigo por la tierra y la organización campesina y, al mismo tiempo, adquiera la capacidad de vincularse al mundo del mercado, creando alternativas de desarrollo al modelo extractivo y de enclave (economía de los pueblos, seguridad alimentaria, los proyectos de finca campesina) (p. 6).

Si bien, se da cuenta de la importancia de la productividad económica para la región, se prioriza el capital social comunitario como elemento trasversal para pensar procesos participativos y equitativos a nivel individual y colectivo. En este sentido, Montero (1984) señala, "que algunos factores psicosociales visibilizan el poder que tienen los individuos para crear soluciones a las dificultades de sus contextos" (p. 390). Así, quienes asumen las posibilidades de cambio, instauran también un compromiso con sus realidades contextuales, dándole mayor validez a la participación y organización colectiva para que sea sostenible y pertinente en el tiempo. Moncayo (1999) afirma que,

El PDPMM ha hecho su mayor apuesta en el desarrollo del capital social, aprender haciendo significa que desde el comienzo los pobladores identifican sus problemas, proponen soluciones, asumen los retos, establecen compromisos, adelanta las acciones, diseñan y aplican los instrumentos para evaluar y ajustar los procedimientos (p. 90).

Es importante hacer mención de la construcción de lo público como escenario donde se fortalecen los vínculos sociales y se piensan alternativas para el alcance de una paz digna, que garantice territorios habitables, donde las comunidades puedan reconocer sus fortalezas y apuesten por reducir las desigualdades 
socioeconómicas; al respecto, según Katz (1999b), "los procesos del PDPMM dan como resultado un modelo de construcción de lo público donde es necesario la afirmación del individuo como sujeto histórico privilegiado, como una forma de organización territorial" (p. 98). Desde esta postura, el desarrollo de una conciencia social colectiva, permitió la emergencia de nuevos posicionamientos de los pobladores, respecto a las decisiones políticas, económicas, sociales y culturales, convirtiéndolos en actores capaces de construir en medio de tensiones y aciertos un movimiento regional por la paz.

Un aspecto característico en el proceso del Programa, es la articulación permanente con las instituciones públicas, buscando construir entre la sociedad y el Estado puentes duraderos y corresponsables en la defensa de los derechos; así, las propuestas debían ser técnicamente viables y políticamente posibles e involucrar en su negociación a los poderes sociales. Articulaciones permanentes, que tienen sus fracturas y recomposiciones de las estrategias que proponen; es así, como lo colectivo, no es solo un lugar armónico, sino lugares de retos, de contrariedades, de relacionamiento intencionales, que no siempre incluye a la totalidad de los sujetos que habitan el territorio.

El fortalecimiento comunitario ha estado permeado de una reconstrucción de la historia, la memoria, la capacidad de escucha, el diálogo, la negociación, la gestión, la mediación de conflictos e intereses que permiten construir un proyecto de vida colectivo. "El fortalecimiento hace hincapié en el aspecto colectivo, en el carácter liberador, en el control y poder centrados en la comunidad y en sus miembros organizados como actores sociales conocedores de su realidad y de los cambios que en ella ocurren" (Montero, 2003, pp. 69-70).

El PDPMM se planteó cuatro enfoques de intervención psicosocial 1. La dimensión individual. 2. De lo individual a lo colectivo. 3. El sujeto colectivo auto reconociéndose como actor social. 4. La configuración del sujeto político en la medida que hay voluntad colectiva para ejercer el poder (PNUD, 2008). Este proceso de intervención tiene su origen, de un lado, en la naturaleza humana de los sujetos individuales y, de otro, en las condiciones colectivas: sociales, históricas, políticas y económicas que constituyen su contexto. Esto, demuestra que los procesos que se generan en el Programa, trascendieron de la exclusión estatal durante muchos años, a la autogestión de una propuesta regional, con unos principios orientadores como la organización, la participación, el empoderamiento y la concertación.

Desde un enfoque de intervención psicosocial, las condiciones anteriormente mencionadas demandan un acompañamiento de reparación y construcción de un escenario de paz desde y para la comunidad. Este compromiso implica que el quehacer psicosocial reconozca unas características particulares de los sujetos y, al mismo, tiempo sus impactos psíquicos, físicos y sociales colectivos. 
Es importante reconocer que las comunidades se encuentran articuladas entre instituciones estatales con intereses diversos, pero es fundamental la visibilización de las organizaciones de bases que tienen una mejor comprensión de la realidad social. En concordancia con los aportes de Baró (1986) "no se trata de que nosotros pensemos por ellos, de que les trasmitamos nuestro esquema o de que les resolvamos sus problemas; se trata de que pensemos y teoricemos con ellos y desde ellos" (p. 297).

Desde la intervención psicosocial, las teorías se construyen a partir de la práctica reflexionada, reconociendo a la intervención psicosocial misma como una aproximación al sujeto en sus capacidades individuales, colectivas y en su contexto histórico, en el que se configuran dinámicas sociales, culturales, políticas y económicas, para construir de manera participativa acciones encaminadas a reflexionar y movilizar necesidades de las comunidades.

Finalmente, se comprende al sujeto como un potencial que en relación con sus pares y otros actores puede agenciar procesos de cambio, pues la comunidad es dinámica y requiere de personas que encaminen su desarrollo al bienestar colectivo; y esto, en gran medida, puede lograrse si se asumen como sujetos políticos, capaces de entender la realidad para una trasformación social.

\section{Conclusiones}

En el acercamiento al Programa de Desarrollo y Paz del Magdalena Medio se resaltaron algunos elementos claves de la intervención psicosocial, partiendo de sus archivos fundacionales y algunas voces académicas que participaron en la construcción comunitaria. Esto develó que la metodología fundacional del Programa, se enmarca en la integralidad, en tanto que comprende las formas de exclusión económica, política y social del Magdalena Medio, vinculando los saberes y los intereses de cada uno de los espacios en donde se significa dicha realidad.

De esta manera, se evidenciaron algunas características psicosociales que emergen dentro de la propuesta y que se gestaron en medio de un contexto de conflicto armado, entendiendo así, que los procesos comunitarios deben fortalecer la participación, el sujeto político, el tejido social y la construcción de lo público en aras de los intereses colectivos. El PDPMM, se puede definir desde sus particularidades como un proceso social en permanente construcción, cambiante, dinámico y sujeto a una recomposición de sí mismo.

La importancia de la articulación del Programa con instituciones públicas y privadas, se reflejó en el fortalecimiento de la administración local y regional, pues condujo a los pobladores a pensar su contexto frente a una planeación 
económica y social, encontrando como mayor reto la movilización de la participación ciudadana y la negociación de intereses internos y externos. El reclamo de la población hacia la débil presencia del Estado, hizo que se dieran convenios entre diferentes gremios para consolidar estructuras solidas que ayudaran a minimizar los diversos problemas sociales, además, gestionó una noción de pertenencia a un sistema político y a un colectivo social.

Se concluye que es de vital importancia conocer y validar las experiencias de construcción de paz en Colombia, en este caso particular el PDPMM que propone, desde una metodología participativa, una alternativa para asumir y vivir un territorio, pensando lo regional y resignificando los procesos generados desde los diferentes actores de la sociedad civil. Es por ello que en la actual coyuntura política del país, todas las experiencias de construcción de paz deben visibilizarse y protegerse mediante mecanismos estatales, que fortalezcan y garanticen un desarrollo acorde con las luchas que durante años se han dado.

Esta aproximación al PDPMM en sus años fundacionales, fue novedosa porque desde allí, se reconoce esta metodología de intervención psicosocial como un aporte significativo, al marco epistémico de construcción de paz por el cual ha transitado el país. Además, porque es una apuesta que desde sus complejidades convoca a ser leídas desde una mirada interdisciplinar, lo cual, es un aporte fundamental para comprender las diversas formas en que las violencias han permeado los territorios y sus pobladores, dando cuenta de los diferentes lugares de enunciación de quienes la han vivido y que pueden pensarse hoy como sujetos políticos. Esto serviría entonces, para rescatar elementos de reflexión tanto prácticos como teóricos, para fortalecer el campo de la intervención psicosocial en Colombia.

\section{Referencias}

Abela, J. A. (2002). Las técnicas de análisis de contenido: una revisión actualizada (Documento de trabajo). Fundación Centro de Estudios Andaluces, España. https://www.centrodeestudiosandaluces.es/publicaciones/ tecnicas-de-analisis-de-contenido-una-revision-actualizada

Baró, M. (1986). Hacia una psicología de la liberación. Boletín de Psicología UCA, 22, 219 -231. http://www.psicosocial.net/historico/index. php?option $=$ com_docman\&view $=$ document $\&$ layout $=$ default $\&$ alias $=320-$ hacia-una-psicologia-de-la-liberacion\&category_slug=psicologia-yviolencia-politica\&Itemid $=100225$ 
Bello, M. N. (Ed.) (2004). Desplazamiento forzado. Dinámicas de guerra, exclusión y desarraigo. Universidad Nacional de Colombia.

Carmona, J. (2009). La carrera de las niñas soldado en Colombia: un estudio desde el punto de vista del agente (Tesis Doctoral). Universidad Complutense de Madrid. https://dialnet.unirioja.es/servlet/tesis? codigo $=144288$

Corporación Juan Bosco (2007). Componente psicosocial. A ritmo de la vida (Documento institucional, inédito). Cali.

De Roux, F. (1996). Documento central de diagnóstico, conclusiones y recomendaciones. Programa de Desarrollo y Paz en el Magdalena Medio, PDPMM, Consorcio SEAP-CINEP, Bogotá.

De Roux, F. (1999). El Magdalena Medio en medio del conflicto y de la esperanza. Revista Controversia, segunda etapa, 1999. Cinep. Bogotá. http:/ / biblioteca.clacso.edu.ar/Colombia/cinep/20100917084007/ ContextoelmagdalenamedioControversiaNo 174.pdf

Díaz, A.; Díaz, J. S. (2015). Qué es lo psicosocial. Ocho pistas para reflexiones e intervenciones psicosociales. En A. Díaz \& E. Moncayo (Ed.). Psicología social crítica e intervención psicosocial, reflexiones y experiencias de investigación (pp. 59-66). Editorial Bonaventuriana.

Díaz-Gómez, Á.; González-Rey, F.; Arias-Cardona, A. M (2017). Pensar el método en los procesos de investigación en subjetividad. CES Psicología, 1O(1), 129-145. https://www.redalyc.org/pdf/4235/423550874009.pdf

Dobles, I. (2009). Ignacio Martín Baró y la psicología de la liberación: un desafío vigente. In M. González (Comp.) Teorías psicosociales (pp. 2007 -230). Universidad de Costa Rica.

Finol, T.; Nava, H. (1999). Procesos y productos de la investigación documental. Editorial de la Universidad del Zulia.

González, F. (2004). O social na psicología e a psicología social: a emergencia do sujeito. Editora Vozes. 
Guerrero, J. P.; Guerrero, L. G.; Herrera, L. Á.; Vargas, M. F. (2011). El Programa de Desarrollo y Paz en el Magdalena Medio. Colombia. Cinep. http://www.desarrollo-alternativo.org/documentos/CINEP.pdf

Katz, M. (1999a). El Proceso metodológico del PDPMM. Revista Controversia, 174, 75-87. http://biblioteca.clacso.edu.ar/Colombia/cinep/20100917092515/EstrategiasdelpdpmmelprocesometodologicodelpdpmmControversiaNo 174.pdf

Katz, M. (1999b). La Construcción de lo público en el escenario actual del Magdalena Medio. Revista Controversia, 174, 96-106. https://core.ac.uk/ download/pdf/35219083.pdf

Moncayo, J. (1999). Los proyectos del PDPMM, un laboratorio de paz. Revista Controversia, 174, 89-95. https://core.ac.uk/download/pdf/35219113.pdf

Montero, M. (1984). La psicología comunitaria: orígenes, principios y fundamentos teóricos. Revista latinoamericana de psicología, 16(3), 387-400. https://www.redalyc.org/pdf/805/80516303.pdf

Montero, M. (2003). Teoría y práctica de la psicología Comunitaria. Paidós.

Montero, M. (2004). Introducción a la psicología comunitaria. Desarrollo, conceptos y procesos. Editorial Paidós.

Programa de las Naciones Unidas para el Desarrollo, PNUD. (2008). Rutas y vivencias de nuestra gente. Sistematización de experiencias en los procesos de los pobladores y pobladoras del Magdalena Medio. http://www.psicosocial.net/ historico $/$ index.php?option $=$ com_docman\&view $=$ download $\&$ alias $=818-$ rutas-y-vivencias-de-nuestra-gente\&category_slug=experiencias-ypropuestas-de-accion $\&$ Itemid $=100225$

Red Nacional de Programas de Desarrollo y Paz, Redprodepez (2016). ¿Qué es un Programa de Desarrollo y Paz, PDP? https://www.redprodepaz.org.co/ que-es-un-pdp-2/

Rodríguez, D. (2017). El genocidio de Ruanda: análisis de los factores que influyeron en el conflicto. Documento Opinión. Instituto Español de Estudios Estratégicos. 
Rodríguez, G.; Gil, F.; García, J. (1996). Metodología de la Investigación Cualitativa. Ed. Aljibe. España.

Observatorio de Paz Integral (2014). Alcance territorial. https://www.opi.org. co/quienes-somos/alcance-territorial

Vargas, M.; Herrera, L.; Guerrero, J.; Guerrero, L. (2010). El programa de desarrollo y paz en el Magdalena Medio. Cinep, 1-14. http://www. desarrollo-alternativo.org/documentos/CINEP.pdf

Villa, J. D. (2012) La acción y el enfoque psicosocial de la intervención en contextos sociales: ¿Podemos pasar de la moda a la precisión teórica, epistemológica y metodológica? El Ágora USB, 12(2), 349-365. http:// www.scielo.org.co/pdf/agor/v12n2/v12n2a05.pdf 


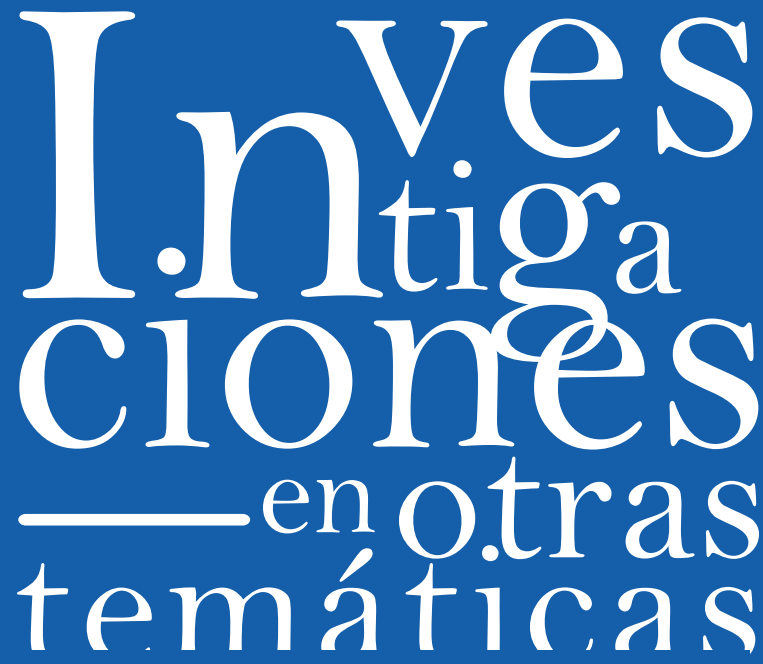

Research on other topics Pesquisa sobre outros tópicos 
